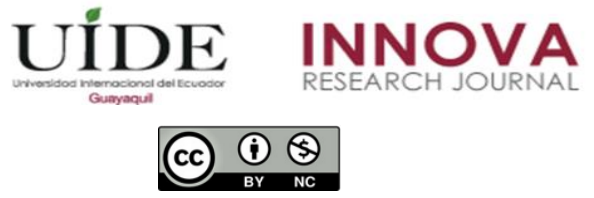

INNOVA Research Journal, ISSN 2477-9024

(Enero-Abril 2020). Vol. 5, No.1 pp. 72-93

DOI: https://doi.org/10.33890/innova.v5.n1.2020.1166

URL: http://revistas.uide.edu.ec/index.php/innova/index

Correo: innova@uide.edu.ec

\title{
Comercio electrónico B2B como estrategia competitiva en el comercio internacional: Desafíos para Ecuador
}

\section{B2B e-commerce as a competitive strategy in international trade: Challenges for Ecuador}

\author{
Allan Elvis Pesántez-Calva \\ Jerry Alexander Romero-Correa \\ Mayiya Lisbeth González-Illescas \\ Universidad Técnica de Machala, Ecuador
}

Autor por correspondencia: aepesantezc_est@utmachala.edu.ec; jromero_est@utmachala.edu.ec; mlgonzalez@utmachala.edu.ec; mmunsibay@gmail.com; lauralinda.cavero@gmail.com; irmamilagros@yahoo.com

Fecha de recepción: 20 de agosto del 2019 - Fecha de aceptación: 28 de octubre del 2019

\section{Resumen}

Este trabajo tiene el propósito de analizar el uso del B2B como herramienta del comercio internacional, bajo el enfoque de estrategia proactiva que favorezca las ventajas competitivas. Se analiza la tipología del comercio electrónico y su evolución en América del Sur y Ecuador. Para el desarrollo de la investigación se utiliza el enfoque cualitativo. Entre los hallazgos se destacan las transformaciones de estructuras de negocio y la necesidad de que en Ecuador se realicen cambios en el ámbito legal, cultura empresarial y sistema de pagos para difundir el uso del B2B. Palabras claves: comercio electrónico; B2B; comercio internacional; comercio electrónico en Ecuador

\begin{abstract}
This paper has the purpose to analyze the use of B2B as a tool of international trade, under the proactive strategy approach that favors competitive advantages. The typology of electronic commerce and its evolution in South America and Ecuador are analyzed. The qualitative approach is used for the development of research. Among the findings are the transformations of business structures and the need for changes in Ecuador to be made in the legal field, business culture and payment system to spread the use of B2B.
\end{abstract}

Key words: e-commerce; B2B; international trade; e-commerce in Ecuador 


\section{Introducción}

Con la aparición del internet muchas de las formas tradicionales de negociación, compra y venta, se han transformado en las empresas. De forma paralela, el uso de la tecnología, particularmente en el proceso de la digitalización de datos, ha impulsado mejoras en la productividad (Aguerre, 2019), atribuídas en parte por la disponibilidad de información y por la velocidad que se requiere para tomar decisiones en mercados de cambios continuos.

El uso de la tecnología se ha vuelto prácticamente un requisito obligatorio a nivel empresarial, sobre todo si de actividades comerciales se trata. Tanta es la repercusión que posee actualmente la terminología "era digital", que varios autores concuerdan en que si una empresa no se adapta a las tendencias actuales, fácilmente terminaría perdiendo su mercado (Tarazona, Medina y Giraldo, 2013; Barrientos, 2017; Costa y Elo, 2018).

El fortalecimiento de las Tecnologías de la Información y Comunicación, en adelante identificadas como TICs, ha contribuido en el desarrollo de un fuerte vínculo entre el comercio y la tecnología en general, permitiendo de esta manera acercarse a un mundo más digitalizado y con acelerados ritmos de crecimiento. Varios autores, afirman que, a partir del desarrollo de estrategias basadas en TICs, las empresas pueden alcanzar un mejor desempeño organizacional (Jones, Motta y Alderete, 2016; Piñeiro, de Llano y Rodríguez, 2016).

El comercio internacional enfrenta grandes retos al estar asociado a mercados que experimentan transformaciones continuas, las cuales se reflejan en la innovación tecnológica y el uso constante de inteligencia artificial alrededor del mundo. Así, el desarrollo de nuevas estructuras de negocios se manifiesta en el uso de tecnologías como el internet, a través de sitios web, aplicaciones y redes sociales. La finalidad principal de dichas estructuras es la obtención de una mayor conectividad, mediante la eliminación de barreras transfronterizas (OMC, 2018).

El comercio electrónico puede ser utilizado como una estrategia para la reducción de costos asociados al transporte, almacenamiento y transacciones en general. Además de permitir mayor rapidez y fiabilidad en las operaciones, ya que disminuye las ineficiencias (Pilinkiene, Kurschus y Auskalnyte, 2013; Jones, Alderete y Motta, 2013).

En Ecuador el uso del comercio electrónico se ha concentrado a nivel local, promoviendo las compras y ventas nacionales, las mismas que se enmarcan en la categoría C2C o B2C. Por otro lado, la compra desde portales extranjeros ha mantenido un crecimiento constante, lo que implica la introducción de productos importados. ProEcuador como institución gubernamental que promueve las exportaciones, ha desplegado programas para incentivar el uso del comercio electrónico B2B. No obstante, las iniciativas del gobierno y el sector empresarial, Ecuador aún debe superar limitaciones asociadas a la confianza de un marco legal escaso, la cultura empresarial para el uso de tecnología y sistema de pagos, entre otros aspectos que caracterizan la problemática para ampliar la utilización del B2B.

Con los argumentos expuestos, este trabajo se guía bajo el propósito de analizar el uso del B2B como herramienta del comercio internacional, bajo el enfoque de estrategia proactiva que favorezca a las empresas ecuatorianas mantener sus ventajas competitivas en escenarios de 
acelerados cambios e innovaciones tecnológicas. Para el desarrollo de la investigación se utiliza el enfoque cualitativo, recurriendo a la revisión de fuentes secundarias y reportes internacionales de instituciones de prestigio internacional.

La estructura del documento presenta la introducción seguida de la revisión de literatura que contempla la evolución del comercio electrónico, los tipos de comercio electrónico B2B, B2C y C2C. Los resultados se presentan con el análisis del comercio electrónico en el mundo, en América Latina, en Ecuador, y las estrategias aplicadas para el desarrollo de comercio electrónico B2B. Finalmente se presentan las conclusiones a manera de implicaciones para Ecuador, se detallan considerando el marco legal, la perspectiva de las empresas y los sistemas de pagos.

\section{Marco Teórico}

\section{Evolución del comercio electrónico}

La forma de comercializar los bienes y servicios ha experimentado grandes cambios generacionales, pasando de medios tradicionales para concretar las operaciones de compra y venta, a la utilización de alternativas tecnológicas que se adaptan a las tendencias del mercado. La principal opción que se presenta en tiempos modernos, es la incursión en el comercio electrónico, aprovechando la globalización de la internet y el flujo de datos transfronterizos (Meltzer, 2018).

El término comercio electrónico, como se lo conoce mundialmente, es definido por varios autores como la actividad que implica la compra-venta de productos o servicios con la particularidad de que en dicho proceso intervienen medios o herramientas tecnológicas, como es el caso de la internet (Sojo, 2001; Kim y Lee, 2016; García, 2018).

El comercio electrónico está basado en el mismo concepto innovador que llegó a implementarse a inicios del año 1920 en negocios del mercado estadounidense, siendo su principal elemento la venta por catálogo, que, además de ser un sistema novedoso para aquella época, significaría un alcance mayoritario de nuevos clientes y por consiguiente un aumento en el número de ventas para las empresas. Dicho sistema de venta se caracterizaba por darle la facilidad al cliente de poder elegir, comprar y recibir los productos desde la comodidad de su hogar (Silva, 2009; Alvarado y Vergara, 2018).

Al igual que cualquier otro concepto novedoso, el comercio electrónico como lo conocemos en la actualidad, ha experimentado cambios de distinta intensidad. En la Tabla 1 recogemos las características que destacan a las etapas diferenciadas en la evolución del comercio electrónico identificadas como generaciones. 
Tabla 1.

Generación del comercio electrónico

\begin{tabular}{|c|c|}
\hline Generaciones & Características \\
\hline Primera generación & $\begin{array}{l}\text { Grandes empresas utilizan páginas web, mostrando contenido general de sus actividades. } \\
\text { No existe compra venta on-line. }\end{array}$ \\
\hline $\begin{array}{l}\text { Segunda } \\
\text { generación }\end{array}$ & $\begin{array}{l}\text { Utilización de páginas web, como canal de venta en la red. } \\
\text { Existencia de múltiples formas de pagos entre ellas convencionales y electrónicas. } \\
\text { Implementación de base de datos. }\end{array}$ \\
\hline Tercera generación & $\begin{array}{l}\text { El pago con tarjeta se vuelve el más común. } \\
\text { Aparición de protocolos para pagos seguros. }\end{array}$ \\
\hline Cuarta generación & $\begin{array}{l}\text { Surgen portales dinámicos que facilitan las compras de acuerdo a preferencias del } \\
\text { consumidor. } \\
\text { Mayor seguridad en intercambio de datos y pagos. }\end{array}$ \\
\hline
\end{tabular}
Adaptado de Seoane (2005)

\section{Tipos de Comercio electrónico}

En términos generales la operatividad del comercio electrónico será siempre la misma, compra-venta de mercancías o servicios a través de canales digitales. Sin embargo, y siguiendo a López y Jouanjean (2017), existen diferentes consideraciones que permiten clasificarlo de la siguiente manera: según la naturaleza (entrega física o digital), según el tipo de flujo (bien o servicio) y según el tipo de transacción (actores que intervienen).

Para los objetivos de nuestra investigación, nos enfocaremos en la clasificación del comercio electrónico según el tipo de transacción. Siguiendo a Turban, King, Liang, Turban y Lee (2015), bajo este criterio, se sitúan operaciones de comercio electrónico, tales como, B2B, B2C, C2B, C2C, G2B, G2C y G2G. Basantes et al. (2016), agregan a dicha lista, transacciones de tipo, B2G, B2I, B2A y C2A. En la tabla 2 sintetizamos los principales tipos de transacciones, identificando los actores que intervienen. 
Tabla 2.

Clasificación de comercio electrónico según el tipo de transacción

\begin{tabular}{ll}
\hline Transacción & Característica \\
\hline B2B & Operación comercial directa entre empresas \\
B2C & Oenta de empresa a consumidor final \\
B2G & Actividad dirigida a captar inversores \\
B2I & Actividad comercial entre empresa y la administración pública \\
B2A & Operación comercial entre consumidores \\
C2C & Actividad comercial entre consumidor y administración \\
C2A & Actividad comercial entre consumidor y negocio \\
C2B & Actividad comercial entre consumidor y gobierno \\
C2G & Operación comercial de gobierno a gobierno \\
G2G & Operación comercial entre gobierno y negocio \\
G2B & Operación comercial de gobierno a consumidor \\
G2C &
\end{tabular}

Adaptado de Basantes et al. (2016)

Es importante aclarar que la clasificación del comercio electrónico según el tipo de transacción, puede variar de acuerdo al criterio de cada autor (Tarazona, Medina y Giraldo, 2013; Ríos, 2014; Gutiérrez, 2015). Sin embargo, y de acuerdo a Moertini (2012) y Sigmond (2018), existen tres tipos que son los más usuales, debido a la participación que presentan en mercados digitales, y son los siguientes: Business to business (B2B), Business to consumer (B2C), Consumer to consumer (C2C).

\section{Comercio electrónico B2B}

El comercio electrónico B2B puede ser definido como la transacción económica efectuada entre empresas que utilizan la internet y diversas tecnologías con la finalidad de conseguir múltiples beneficios, entre los cuales podemos mencionar los siguientes: mayor eficiencia, aumento de ventas, mejoras en las relaciones con los clientes y mayor penetración en el mercado (Teo y Ranganathan, 2004; Ballesteros y Ballesteros, 2007 y Sila, 2015).

\section{Comercio electrónico B2C}

Laudon y Guercio (2017) definen al comercio electrónico B2C como el tipo de negocio en línea en donde las empresas, intentan llegar a un consumidor individual. Este tipo de comercio electrónico se caracteriza principalmente por la compra de bienes al por menor. Aunque el B2C es muy conocido y aplicado a nivel mundial, los autores antes mencionados, estiman que con el tiempo, su participación disminuirá notablemente. 


\section{Comercio electrónico C2C}

El comercio electrónico $\mathrm{C} 2 \mathrm{C}$ es definido como una versión moderna, derivada de los tipos de comercio en línea ya existentes. Se caracteriza por interacciones comerciales, efectuadas entre consumidores, generalmente a través de medios sociales alojados en la internet. Debido a su fácil acceso y reducidos costos de aplicación es mayoritariamente utilizado por pequeños comerciantes y grandes minoristas (Sukrat, Mahatanankoon y Papasratorn, 2016; UNCTAD, 2016; Leonard y Jones, 2019).

\section{Metodología}

Esta investigación se realiza bajo un enfoque cualitativo. Se considera para el cumplimiento de los objetivos planteados, la aplicación de métodos teóricos. El método histórico lógico se emplea para identificar la evolución del comercio electrónico. El método analíticosintético y el inductivo deductivo son útiles para la revisión teórica de los conceptos asociados, el análisis de las estrategias empleadas y el estado de implementación del B2B en el mundo, América Latina y Ecuador.

A partir de la revisión de literatura relacionada a nuestro objeto de estudio, el comercio electrónico de tipo B2B, realizamos una exploración de trabajos actualizados publicados por otros autores en revistas científicas ampliamente reconocidas en el campo académico. Además, se ha identificado evidencia empírica procedente de informes internacionales sobre la evolución del comercio electrónico, cuyas fuentes institucionales gozan de prestigio.

\section{Resultados}

Con las premisas teóricas expuestas, los resultados se presentan como el análisis de la situación del comercio electrónico en el contexto mundial, América Latina, para finalmente enfocarnos en el nivel de desarrollo del comercio electrónico en Ecuador. En el marco de los objetivos de esta investigación, nuestro interés se centra en la tipología de comercio electrónico $\mathrm{B} 2 \mathrm{~B}$, la misma que se refiere a la relación comercial directa entre empresas.

Considerando la necesidad de las empresas exportadoras de defender su posición en mercados altamente competitivos, la aplicación del B2B aumenta las posibilidades de fortalecer el desempeño comercial, a través de mejorar las relaciones con los clientes, la comunicación, el intercambio de información (Biemans, Brenčič, \& Malshe, 2010). La colaboración entre la empresa exportadora y su cliente, puede conducir a una mejor comprensión de las necesidades mutuas en la negociación, requisitos y procesos. El B2B puede robustecer el aprendizaje organizacional, por ejemplo, a partir del intercambio de información, la empresa exportadora podría organizar las adaptaciones que requiere para cubrir las necesidades del cliente.

Finalmente, y no menos importante, el B2B puede ampliar las posibilidades para mejorar la dirección del conocimiento en las empresas, estrategia a través de la cual se orienta hacia un mejor desempeño organizacional en ambientes de mercados dinámicos (Claver et al., 2017a; Claver et al., 2017b). 


\section{Comercio electrónico en el mundo}

Datos proporcionados por la UNCTAD (2017) señalan que a nivel mundial las operaciones vinculadas al comercio electrónico lograron conseguir un ascenso muy cercano a los 26 billones de dólares. En la tabla 3 se detallan los principales países, que poseen mayor participación en comercio electrónico a nivel mundial.

Tabla 3.

Países con mayor participación en comercio electrónico a nivel mundial

\begin{tabular}{ccc}
\hline País & $\begin{array}{c}\text { Porcentaje de participación en } \\
\text { e-commerce }\end{array}$ & $\begin{array}{c}\text { Valor en billones de dólares } \\
\text { americanos }\end{array}$ \\
\hline Estados Unidos & $28 \%$ & 7,10 \\
Japón & $10 \%$ & 2,53 \\
China & $8 \%$ & 2,02 \\
República de Corea & $5 \%$ & 1,30 \\
Alemania & $4 \%$ & 1,01 \\
Resto del mundo & $45 \%$ & 11,39 \\
\hline
\end{tabular}

Adaptado de UNCTAD (2017)

Países como Estados Unidos, Japón y China, lideran el comercio electrónico a nivel mundial, sin embargo, esto no significa que todas sus transacciones están relacionadas al B2B, por este motivo es fundamental para nuestra investigación identificar de manera específica, transacciones de la tipología antes mencionada. En la tabla 4 se presentan los valores generados a nivel mundial por comercio electrónico B2B. 
Tabla 4.

Países con mayor participación en comercio electrónico B2B

\begin{tabular}{ccc}
\hline País & $\begin{array}{c}\text { Participación en comercio } \\
\text { electrónico B2B, proporcional a } \\
\text { su actividad total }\end{array}$ & $\begin{array}{c}\text { Valor en billones de dólares } \\
\text { americanos }\end{array}$ \\
\hline República de Corea & $96 \%$ & 1,1 \\
Japón & $96 \%$ & 2,4 \\
Estados Unidos & $91 \%$ & 6,4 \\
Alemania & $91 \%$ & 0,9 \\
Canadá & $90 \%$ & 0,4 \\
España & $90 \%$ & 0,2 \\
Francia & $89 \%$ & 0,5 \\
Australia & $87 \%$ & 0,1 \\
Reino Unido & $76 \%$ & 0,6 \\
China & $69 \%$ & 1,3 \\
\hline
\end{tabular}

Adaptado de UNCTAD (2017)

El caso de China es bastante particular, pese a formar parte de las tres economías con mayor actividad en comercio electrónico, el país oriental se ubica en el décimo puesto frente a transacciones direccionadas al B2B en el entorno mundial. Sin embargo, debido al gran volumen de ventas que registra, termina generando valores monetarios mucho más altos, en comparación a economías que poseen mayor participación en aplicación de transacciones B2B.

En términos de comercio electrónico, las transacciones basadas en B2B representan el $89 \%$ a nivel mundial, de dicho porcentaje un $64 \%$ corresponde a las 10 principales economías del mundo que efectúan operaciones de comercio electrónico (Ueasangkomsate, 2015; UNCTAD, 2017). Mencionadas cifras, convierten al B2B en la tipología electrónica comercial de mayor aplicación en el mundo.

Un estudio realizado por Mehta y Hamke (2019) revela que el comercio electrónico B2B, generó una cifra muy cercana a los 10,6 billones de dólares en el año 2018. Además, afirma que mercados asiáticos, seguirán liderando las actividades de comercio electrónico B2B con una participación aproximada del 80\% a nivel mundial. Debido a que el comercio electrónico B2B genera operaciones más eficaces, Alsaad, Mohamad, Taamneh e Ismail (2018) indican, que este tipo de comercio se extenderá notablemente, sobre todo, en economías altamente competitivas.

\section{Comercio electrónico en América Latina}

Mercados en etapas de desarrollo, como los países de América Latina y el Caribe, deben enfrentarse a varios desafíos particulares que se sitúan a nivel interno de cada país. Muchos de estos desafíos son denominados por Zwillenberg, Field y Dean (2014) como "fuentes de fricción”, y están relacionados en la mayoría de los casos con la infraestructura, difícil acceso a 
tecnología, costos y regulaciones prácticamente obsoletas, sumándose a dicha lista, la escasa o nula capacitación en temas relacionados al uso óptimo de las TICs.

Centrándonos en instrumentos legales de regulación internacional, podemos afirmar que es complejo encontrar una normativa que garantice operaciones relacionadas al comercio electrónico en su totalidad. Si bien es cierto, en organismos de alcance internacional como la Organización Mundial del Comercio (OMC) se han tratado ciertos aspectos superficiales en su normativa, hasta la actualidad no se han implementado reformas directamente vinculadas al comercio digital, tomando en cuenta que dichas normativas se implementaron incluso antes de la globalización de la internet (Meltzer, 2018).

Uno de los grandes problemas que se puede evidenciar en América Latina es la falta de una política comercial integral. La inexistencia de la misma ha generado múltiples barreras comerciales, que con el tiempo se han convertido en el factor principal de un escaso dinamismo comercial, tanto tradicional como electrónico.

Meltzer (2018) afirma que es fundamental que la región de América Latina y el Caribe implementen una política de comercio integrado, de esta manera se aprovecharía al máximo cuestiones direccionadas al comercio electrónico internacional. Señala además que dicha política sería clave para un proceso de transformación. En la figura 1 se describen los beneficios que traería consigo la implementación de sistemas de comercio electrónico en los países de la región.

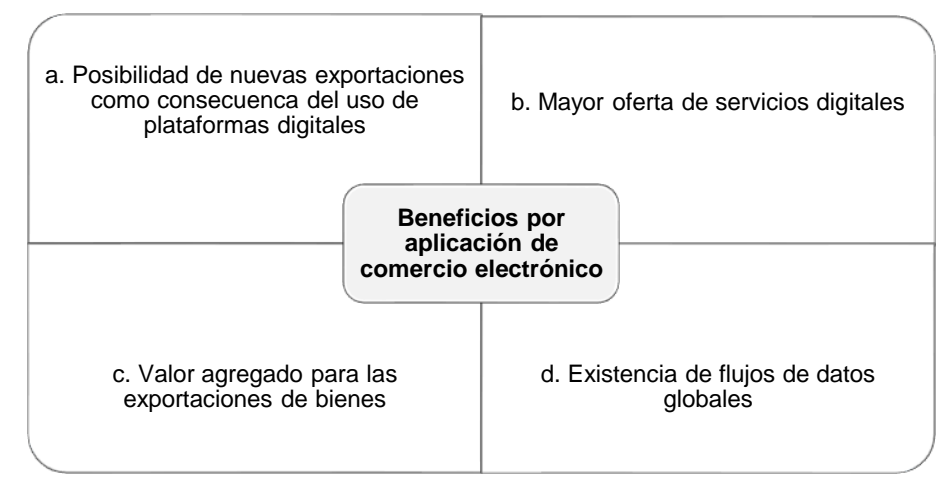

Figura 1. Beneficios por la implementación de sistemas de comercio electrónico en América Latina y El Caribe. Adaptado de Meltzer (2018)

A pesar de que podría calificarse al comercio electrónico como un proceso lento en la región, sería ilógico pensar que América Latina no forma parte de la transformación y revolución digital que se está generando. Siguiendo las conclusiones del trabajo de Suominen (2017), más de la mitad de la región está conectada al internet actualmente. Este dato refleja un incremento significativo, tomando en cuenta que, en el año 2000, únicamente el $5 \%$ de la población estaba conectada.

El acelerado crecimiento que presentan las actividades vinculadas al comercio electrónico a nivel mundial, está haciendo que la región cambie de manera notable los patrones tradicionales de comercialización, optando por alternativas basadas en mejoras tecnológicas, que brinden mayor visibilidad en mercados internacionales (Broome, 2016). 
Para Gómez (2018), la región de América Latina no ha explotado en su totalidad el comercio electrónico debido a que se encuentra en una etapa muy temprana de desarrollo, impidiendo así un potencial crecimiento.

En la actualidad, los países que muestran mayor actividad de comercio electrónico en la región de América Latina y el Caribe son: Brasil, Chile, Colombia, México y Perú. Por su parte países como Ecuador, Paraguay, Venezuela y Bolivia, registran los índices más bajos. En cuanto a nivel de empresas, un $51 \%$ de empresas grandes de la región tienen participación en el comercio electrónico, esto incluye tanto compras como ventas (CEPAL, 2018).

Obtener estadísticas oficiales del comercio electrónico B2B a nivel internacional, es uno de los principales obstáculos que se presentan en investigaciones relacionadas a estos temas. Las razones que derivan de lo antes expuesto, se asocian a la dificultad de acceso a los datos o la no existencia de los mismos. En el caso específico de América Latina, países como Argentina, Brasil y Chile, utilizan información recopilada por cámaras de comercio y empresas privadas. Es preciso acotar que la información que registran dichas instituciones, no es de libre acceso (UNCTAD, 2017 y CEPAL, 2018).

\section{Comercio electrónico en Ecuador}

Ecuador se enfrenta al desafío de fomentar el uso de las TICs en el comercio a nivel doméstico y en el ámbito de los negocios internacionales, particularmente en las empresas exportadoras. Varias iniciativas para impulsar el comercio electrónico se han identificado por parte del Gobierno y empresas vinculadas al comercio electrónico, intentando dar un paso de lo tradicional a lo digital, sin embargo, muchos de estos han fracasado. Un claro ejemplo de lo antes mencionado, se pudo evidenciar con la introducción del dinero electrónico en Ecuador, el mismo que estuvo vigente por un poco más de tres años, para luego desaparecer sin haber cumplido las expectativas planteadas de inclusión financiera (Campuzano, Chávez y Maza, 2018). La baja aceptación por parte de los usuarios, debido a la desconfianza, fue uno de los principales factores, que impidió el normal desarrollo de este nuevo sistema. Se estima que aproximadamente un $71 \%$ de cuentas que se activaron en un inicio para su utilización, se encontraban inactivas (El Universo, 2017).

Instituciones como la Cámara Ecuatoriana de Comercio Electrónico (CECE) en conjunto con el Ecommerce Institute, han efectuado eventos como el denominado Ecommerce Day Ecuador, con el objetivo de divulgar la importancia y beneficios que brinda la economía digital a nivel de empresas, creando de esta manera cierto nivel de reflexión en relación al poder e impacto que representan la internet y las nuevas tecnologías en el mercado actual (Ecommerce Day, 2018). Cabe destacar que dicho evento, bajo la denominación "Ecommerce Day" se lleva a cabo en diferentes países de América Latina, siendo una iniciativa propia del Ecommerce Institute.

En aspectos relacionados a la normativa, es importante mencionar que se han integrado cuestiones directas al comercio electrónico en el nuevo "Código de Comercio", vigente a partir de mayo del 2019. Anteriormente el único instrumento legal disponible era la "Ley de Comercio Electrónico, Firmas Electrónicas y Mensajes de Datos”, éste último mostrando algunas 
deficiencias, debido a que fue elaborado en el año 2002. Según el Informe sobre el Comercio y el Desarrollo emitido por la UNCTAD (2018), la regulación de la comercialización digital, es un aspecto clave en el que deberían preocuparse los países en desarrollo.

Ecuador ha experimentado un notable crecimiento en relación al uso del internet en los últimos años, llegando a ocupar el quinto puesto a nivel de Sur América en los países con mayor uso de red. Un equivalente al 79,9 \% en la región, según estadísticas del Internet World State (2018).

A pesar de los registros positivos que Ecuador ha mostrado en los últimos periodos con actividades vinculadas al comercio electrónico, tal como lo reflejan estadísticas de la Superintendencia de Bancos (2018), éstas han sido en el mejor de los escenarios a nivel local, es decir compras y ventas domésticas, las mismas que en la mayoría de los casos, podríamos denominar de categoría C2C o B2C, ya que por lo general son operaciones comerciales entre consumidores o entre empresa y consumidor final. En la tabla 5 se detalla la actividad que ha tenido Ecuador en el comercio electrónico, tanto en portales nacionales como internacionales.

Tabla 5.

Comercio electrónico en Ecuador

\begin{tabular}{ccc}
\hline Año & $\begin{array}{c}\text { Porcentaje de compra-venta en } \\
\text { portales nacionales }\end{array}$ & $\begin{array}{c}\text { Porcentaje de compra en portales } \\
\text { extranjeros }\end{array}$ \\
\hline 2016 & $16,70 \%$ & $83,27 \%$ \\
2017 & $12,61 \%$ & $87,37 \%$ \\
2018 & $12,70 \%$ & $87,28 \%$ \\
\hline
\end{tabular}

Adaptado de Observatorio de Comercio Electrónico de la Universidad Espíritu Santo (2018)

Partiendo de la información presentada en la tabla 5, se puede inferir que el comercio electrónico de Ecuador gira entorno a compras en portales extranjeros, sobresaliendo entonces transacciones de tipología C2C y B2C, que si bien es cierto, ayudan al fortalecimiento de un sistema comercial basado en las TICs, es muy poco probable que generen beneficios directos a nivel de país. Tomando en consideración que, en dichas tipologías de comercio electrónico, generalmente intervienen mercancías importadas, se desaprovecha la oportunidad de utilizar el comercio electrónico como herramienta orientada a la exportación de productos nacionales.

Encontrarse con tiendas virtuales o empresas con presencia en la web, no es un tema nuevo para Ecuador, ya que se estima que un alrededor de 700 tiendas online, existen en el país (El Comercio, 2019). Este escenario es muy distinto al identificar instituciones que brinden servicios orientados al desarrollo de negocios en línea, en donde se evidencia una escasa existencia. En la tabla 6 se detallan las instituciones y empresas más conocidas que brindan los servicios antes mencionados. 
Tabla 6.

Instituciones y empresas vinculadas al desarrollo de comercio electrónico en Ecuador

\begin{tabular}{|c|c|c|c|c|}
\hline $\begin{array}{l}\text { Nombre de } \\
\text { institución o } \\
\text { empresa }\end{array}$ & Sector & Actividad & Ventajas & Desventajas \\
\hline $\begin{array}{l}\text { Cámara Ecuatoriana } \\
\text { de Comercio } \\
\text { Electrónico }\end{array}$ & Privado & $\begin{array}{l}\text { Brindar capacitaciones, } \\
\text { certificaciones, sellos de } \\
\text { confianza. }\end{array}$ & $\begin{array}{l}\text { Respaldo de Asociación } \\
\text { ecuatoriana de software } \\
\text { Entre sus planes está la } \\
\text { asistencia en búsqueda de } \\
\text { apoyo por parte del } \\
\text { gobierno. }\end{array}$ & $\begin{array}{l}\text { Algunas de sus } \\
\text { actividades no se } \\
\text { encuentran disponibles } \\
\text { actualmente. }\end{array}$ \\
\hline Negos Holding & Privado & $\begin{array}{l}\text { Desarrollar negocios en } \\
\text { línea. } \\
\text { Terminales de puntos de } \\
\text { venta, facturación } \\
\text { electrónica y botones de } \\
\text { pagos. }\end{array}$ & $\begin{array}{l}\text { Aliado de la Cámara de } \\
\text { Comercio de Quito } \\
\text { Asesorías y seguimiento } \\
\text { de ventas. } \\
\text { Apoyo en logística. }\end{array}$ & \\
\hline E-consulting $360^{\circ}$ & Privado & $\begin{array}{l}\text { Asesorías en estrategias de } \\
\text { ventas electrónicas. } \\
\text { Desarrollo Web } \\
\text { Consultora en Marketing } \\
\text { Digital }\end{array}$ & $\begin{array}{l}\text { Respaldo de Asociación } \\
\text { ecuatoriana de software } \\
\text { Respaldo de Cámara } \\
\text { ecuatoriana de Comercio } \\
\text { Electrónico }\end{array}$ & $\begin{array}{l}\text { No cuentan con apoyo } \\
\text { de un organismo } \\
\text { público. }\end{array}$ \\
\hline
\end{tabular}

Elaborado por los autores

Es importante aclarar, que en Ecuador existen varias empresas que brindan servicios relacionados a la creación de tiendas virtuales, sin embargo, no han sido consideradas en la tabla anterior, ya que sus actividades se limitan al desarrollo de un sitio web.

Analizando brevemente la información expuesta en párrafos anteriores, hasta cierto punto se podría considerar que el comercio electrónico en territorio ecuatoriano, se viene desarrollando de una manera aceptable. Sin embargo, surge la pregunta ¿Se ha utilizado el comercio electrónico como herramienta para obtener beneficios en el ámbito internacional?

Para poder responder la pregunta planteada, es necesario mencionar a ProEcuador, institución que actualmente se encarga de actividades vinculadas al comercio electrónico a nivel internacional, debido a que, en sus funciones principales, se encuentra la ejecución de políticas y normas de promoción para exportaciones del Ecuador.

ProEcuador en el uso de sus funciones y reconociendo la importancia de utilizar la tecnología como aliada para el proceso de internacionalización de las empresas, ha venido trabajando en los últimos años, en iniciativas basadas en herramientas digitales, las mismas que están enfocadas en potencializar la promoción y comercialización de productos nacionales, en mercados extranjeros (Ministerio de Comercio Exterior e Inversiones, 2016). La principal alternativa, lograr implementar sistemas de comercialización electrónica B2B. 
En el año 2018, con el apoyo de FromOzz, empresa especialista en comercio electrónico, ProEcuador consigue lanzar el primer portal B2B del Ecuador desarrollado por una institución pública, cuya finalidad era promocionar la oferta exportable de empresas ecuatorianas (El Universo, 2018). El nombre con el que se identifica a la plataforma es e-Ecuador, la misma que presenta actualmente un total de 2 sectores de exportación, el primero orientado a productos, en donde existen 16 categorías y un segundo sector, enfocado a servicios, conformado por dos apartados.

La implementación de e-Ecuador, representa sin duda un avance significativo para el comercio electrónico ecuatoriano, tomando en cuenta que transacciones de tipo B2B, son las que brindan beneficios directos al desarrollo digital y la economía de un país.

Para poder acceder a los beneficios de e-Ecuador, es necesario que la persona o empresa esté registrada como usuaria ProEcuador, de lo contrario y posterior a su solicitud de registro deberá ser capacitada y pasar por la metodología denominada "Ruta del exportador", un sistema utilizado para dar clasificación a los usuarios, de acuerdo a criterios establecidos por dicha institución. Actualmente e-Ecuador, registra un total de 1866 empresas exportadoras y 382 compradores a nivel mundial (El Telégrafo, 2019).

Ecuador, no es el primer país en la región que ha optado por iniciativas basadas en plataformas electrónicas B2B. Brasil, Chile y Colombia ya lo han hecho. En el caso particular de Brasil, la plataforma cuenta con dos versiones, una en portugués y otra totalmente en español, con la finalidad de poder obtener un mayor acceso a mercados latinos. Chile por su parte, pide como requisitos para poder ser parte de su plataforma, que la empresa haya registrado exportaciones por un monto de US \$ 40.000 en bienes o US $\$ 10.000$ en el caso de servicios. Dichas cifras deben corresponder a operaciones realizadas durante el periodo 2016-2018, según se expresa en el portal ProChile (2019).

El portal web El Telégrafo (2019) afirma que e-Ecuador, hasta finales del año 2018, logró generar un total de $\$ 2$ '000.000 a partir de negocios concretados. Sin embargo, actualmente recopilar datos reales de la participación que ha tenido Ecuador, en comercio electrónico a nivel internacional, es muy complicado. El panorama es idéntico al presentado por varios países de la región, con la diferencia de que, en muchos informes realizados por organismos internacionales, Ecuador, ni siquiera es mencionado.

Ahora bien, si Ecuador ha utilizado sistemas de comercio electrónico basados en B2B, ¿Por qué no forma parte de las estadísticas internacionales? En primer lugar, hay que tener en cuenta que el uso de plataformas orientadas al B2B en Ecuador, es algo prácticamente nuevo, y lograr desarrollarlas, representa varios desafíos. Por otro lado, es importante mencionar que a pesar de la iniciativa desarrolla por ProEcuador, la aplicación de comercio electrónico B2B, no se puede limitar, a la adopción por parte de entidades gubernamentales. Empresas privadas con gran potencial de oferta exportable, podrían ocupar estas nuevas herramientas con la finalidad de extender sus destinos comerciales. 
Es lógico pensar que algunas empresas ecuatorianas muestren desconfianza a sistemas basados en comercio electrónico, muchas veces ocasionados por el desconocimiento o por el simple temor de enfrentarse a nuevos retos, desaprovechando así, herramientas de gran utilidad.

Suominen (2017), en su estudio realizado para Latin America's Late Digital Ecosystem, detalla que una empresa que no tenga operaciones asociadas a la internet, estaría perdiendo al menos un $15 \%$ de su productividad. Señala además, que la presencia online de las empresas ha facilitado conseguir nuevos clientes en el exterior, incluso en mercados a los que no se llegaba con sistemas tradicionales de venta. Por su parte, autores como Abebe (2014); Chong, Bian y Zhang (2015) y Winwin (2019), aseguran que la adopción del comercio electrónico, afecta positivamente al promedio de ventas e incrementa la competitividad en el mercado.

Se presenta entonces, un nuevo escenario. Las empresas desean aprovechar los beneficios que genera el comercio electrónico B2B, sin embargo se encuentran con varios problemas que no permiten un normal desarrollo de actividades comerciales en la web. En la tabla 7 se describen varios problemas con la posible causa asociada a su origen.

Tabla 7.

Problemas al implementar sistemas de comercio electrónico B2B

\begin{tabular}{cl}
\hline Problema & Origen \\
\hline Cultura tecnológica & $\begin{array}{c}\text { Empresas que siguen utilizando métodos } \\
\text { tradicionales de ventas. } \\
\text { Sistemas internos en empresas }\end{array}$ \\
$\begin{array}{c}\text { Creación de sistemas empresariales que no están } \\
\text { orientadas al uso de tecnología. }\end{array}$ \\
Coste \\
$\begin{array}{c}\text { Algunas empresas no son capaces de implementar } \\
\text { sistemas de comercio electrónico B2B, debido a la } \\
\text { inversión que se requiere. }\end{array}$ \\
Oferta no adoptada \\
$\begin{array}{c}\text { En ocasiones las empresas no toman en cuenta que su } \\
\text { cliente es otra empresa y definen mal su oferta. }\end{array}$
\end{tabular}

Adaptado de Sánchez (2019)

Autores como Kshetri y Dholakia (2002), identifican como problemas de adopción al comercio electrónico B2B, factores internos a nivel de país, entre los que se encuentran los siguientes: tamaño de población, capital empresarial, infraestructura en telecomunicaciones, aspectos logísticos, impuestos o incentivos, barreras arancelarias, regulaciones y situación política.

En el caso específico de Ecuador, se pueden identificar varios obstáculos que impiden el desarrollo del comercio electrónico con alcance internacional. En la tabla 8 se describen los principales factores que limitan la aplicación y obtención de beneficios a partir de comercio electrónico B2B. 
Tabla 8.

Obstáculos a los que se enfrenta Ecuador para el desarrollo de comercio electrónico B2B.

\begin{tabular}{ll}
\multicolumn{1}{c}{ A nivel país - empresarial } & \multicolumn{1}{c}{ A nivel internacional } \\
\hline Inexistencia de una entidad estatal, encargada & $\begin{array}{l}\text { Inexistencia de una política de comercio } \\
\text { únicamente del manejo de operaciones en } \\
\text { comercio electrónico. }\end{array}$ \\
$\begin{array}{l}\text { Caribe. } \\
\text { Utilización de normativa desactualizada. }\end{array}$ & $\begin{array}{l}\text { Inexistencia de una normativa específica para } \\
\text { reglamentar operaciones de comercio electrónico. }\end{array}$
\end{tabular}

Falta de capacitación en uso de tecnologías orientadas al comercio.

Altos costos por envíos transfronterizos.

Cultura tecnológica no desarrollada.

Problemas logísticos.

Altos costos en la implementación de sistemas de pagos seguros.

Tiempos de envíos de mercancías demorados.

\section{Elaborado por los autores}

Varios de los obstáculos mencionados en la Tabla 8, no son exclusivos de Ecuador. En gran parte de América Latina y El Caribe, el panorama es prácticamente igual. Por lo antes mencionado, es preciso acotar que Ecuador podría aprovechar dicha realidad y lograr obtener una ventaja competitiva en relación a sus vecinos regionales, siempre y cuando logre superar la problemática actual.

\section{Estrategias aplicadas para el desarrollo de comercio electrónico B2B}

Con la finalidad de obtener los verdaderos beneficios del comercio electrónico B2B, varios países a nivel mundial han optado por la implementación de estrategias que ayuden al crecimiento y desarrollo de economías basadas en sistemas de comercialización digital. En la tabla 9 se describen los estudios de varios autores que se recogen las estrategias asociadas al comercio electrónico empleadas por empresas de diferentes sectores y país de origen. 
Tabla 9.

Estrategias aplicadas en varios países para el fortalecimiento del comercio electrónico.

\begin{tabular}{|c|c|c|c|}
\hline Países & Autor & Ámbito de estudio & Estrategias \\
\hline India & $\begin{array}{l}\text { Chaudhuri y } \\
\text { Kumar } \\
\text { (2015) }\end{array}$ & $\begin{array}{ll}\text { Empresas } & \text { de } \\
\text { manufacturas } & \\
\text { Puertos gestión de } \\
\text { bunkers }\end{array}$ & $\begin{array}{l}\text { Inclusión de sistemas de seguimientos satelital } \\
\text { global, digitalización de puertos y red de } \\
\text { mercados internacionales. }\end{array}$ \\
\hline \multirow[b]{2}{*}{$\begin{array}{l}\text { Estados } \\
\text { Unidos }\end{array}$} & Sila (2015) & $\begin{array}{l}197 \text { casos de estudios } \\
\text { empíricos }\end{array}$ & $\begin{array}{l}\text { Aplicación de estrategias operativas y } \\
\text { competitivas, desarrolladas en cuatro ámbitos: } \\
\text { factor organizacional, factor tecnológico, factor } \\
\text { inter organizacional y factor ambiental. }\end{array}$ \\
\hline & $\begin{array}{l}\text { Hamad, } \\
\text { Elbeltagi, } \\
\text { Jones y El- } \\
\text { Gohary } \\
(2015)\end{array}$ & Empresas manufactureras & $\begin{array}{l}\text { Aumento de políticas enfocadas al desarrollo de } \\
\text { pequeñas y medianas empresas a través de } \\
\text { tecnología, estructuración organizacional y } \\
\text { ambiental }\end{array}$ \\
\hline \multirow[b]{2}{*}{ China } & $\begin{array}{l}\text { Chen, Chen } \\
\text { y Lan }(2016)\end{array}$ & Industria de redes & $\begin{array}{l}\text { Mejora continua en servicio de información, } \\
\text { transacción e integración de recursos. }\end{array}$ \\
\hline & $\begin{array}{l}\text { HaiBo, Juan } \\
\text { y Jie (2016) }\end{array}$ & $\begin{array}{l}\text { Encuestas a } 384 \\
\text { industrias, respecto a } \\
\text { evaluación de calidad en } \\
\text { sitios de comercio } \\
\text { electrónico }\end{array}$ & $\begin{array}{l}\text { Innovación: comercio electrónico + marketing } \\
\text { digital + infraestructura. }\end{array}$ \\
\hline Ucrania & $\begin{array}{l}\text { Zatonatska y } \\
\text { Kornieieva } \\
\text { (2016) }\end{array}$ & $\begin{array}{l}\text { Evaluación por parte del } \\
\text { gobierno a empresas, en } \\
\text { Ucrania }\end{array}$ & $\begin{array}{l}\text { Incremento de seguridad de la internet, } \\
\text { distribución de tarjetas de crédito, calidad de } \\
\text { servicios postales. } \\
\text { Superar impuestos de pagos y desarrollo de } \\
\text { software. }\end{array}$ \\
\hline Bangladesh & $\begin{array}{l}\text { Karim y Qi } \\
(2018)\end{array}$ & $\begin{array}{l}\text { Recolección de datos a } \\
\text { partir de consumidores } \\
\text { locales }\end{array}$ & $\begin{array}{l}\text { Adecuación de pagos a través de tarjetas de } \\
\text { créditos internacionales, inclusión de sistema } \\
\text { logístico de transporte, políticas gubernamentales } \\
\text { e integración de redes sociales con plataformas } \\
\text { del comercio electrónico. }\end{array}$ \\
\hline Vietnam & $\begin{array}{l}\text { EU-Vietnam } \\
\text { Business } \\
\text { Network } \\
(2018)\end{array}$ & $\begin{array}{l}\text { Canales de puertos } \\
\text { (Viettel, EMS, VNPost, } \\
\text { Supership, } \\
\text { Giaohangtietkiem) y } 600 \\
\text { exportadores vietnamitas }\end{array}$ & $\begin{array}{l}\text { Eliminación de derechos de aduana para } \\
\text { negociaciones electrónicas, con la finalidad de } \\
\text { incrementar oportunidades comerciales. }\end{array}$ \\
\hline $\begin{array}{l}\text { Croacia y } \\
\text { Turquía }\end{array}$ & $\begin{array}{l}\text { Erceg y Kilic } \\
(2018)\end{array}$ & $\begin{array}{l}\text { Logística empresarial en } \\
\text { comercio electrónico de } \\
\text { negocios locales }\end{array}$ & $\begin{array}{l}\text { Turquía: eliminación a límites de mercados } \\
\text { mediante lazos comerciales. } \\
\text { Croacia: implementación de sistemas logísticos } \\
\text { electrónicos, donde el cliente puede probar el } \\
\text { producto para su posterior aceptación o } \\
\text { devolución. }\end{array}$ \\
\hline
\end{tabular}

Elaborado por los autores

Ecuador podría tomar como referencia varias de las estrategias que han sido aplicadas por diferentes países y empezar a trabajar en el fortalecimiento y desarrollo de una economía basada en las TICs. De esta manera se aprovecharía al máximo la implementación de tecnología en las diferentes empresas del país y se generaría una ventaja con respecto a los países de la región. 


\section{Conclusiones}

Luego de haber realizado un análisis de la evolución del comercio electrónico, desde sus diferentes etapas y desarrollo en varios países, con énfasis en América Latina, podemos manifestar lo siguiente:

El desarrollo de nuevas tecnologías ha permitido que el comercio electrónico, sea adoptado por las empresas, como un nuevo modelo de negocio, debido a las grandes ventajas que puede llegar a brindar. Alcanzar un mejor desempeño organizacional, disminuir las ineficiencias, conseguir mayor conectividad con el mercado extranjero, no perder productividad, agilizar los procesos de negociación e incrementar las ventas, han sido sin lugar a duda los principales factores, que hacen del comercio electrónico una herramienta de potencial crecimiento en el ámbito empresarial.

Actualmente en Ecuador las transacciones derivadas de comercio electrónico con mayor participación son de tipo, C2C y B2C. Por la iniciativa de instituciones como ProEcuador, se ha venido trabajando en la aplicación de plataformas electrónicas orientadas al B2B, con la finalidad de obtener beneficios directos para el país.

Existen múltiples desafíos que Ecuador debe enfrentar para poder lograr desarrollar una economía basada en sistemas de comercialización electrónica B2B. A continuación, se describen algunas alternativas que el país debería tomar a consideración en diferentes ámbitos:

\section{a) Marco legal}

A pesar de haber incluido al comercio electrónico en el nuevo "Código de Comercio" es necesaria una actualización a la "Ley de Comercio Electrónico, Firmas Electrónicas y Mensajes de Datos", ya que fue elaborada en el año 2002 y presenta varias deficiencias, tomando en consideración que el comercio electrónico ha venido evolucionando constantemente.

b) A nivel de empresas

La no existencia de una cultura tecnológica desarrollada en el país, impide que muchas empresas se adapten a los cambios digitales que presenta el mercado actual. Es importante que las empresas empiecen por un cambio organizacional que permita mejorar los procesos de negocios, a través de la innovación tecnológica.

Entidades gubernamentales podrían crear iniciativas o programas, que permitan a las organizaciones conocer la importancia de sustituir los patrones tradicionales de negociación y venta.

c) Sistemas de pago

Implementar sistemas de pagos seguros en una plataforma de venta electrónica, es sin duda un problema para las empresas ecuatorianas, esto debido a los altos porcentajes que representa su aplicación. Actualmente el uso de un botón de pago seguro, le significa a la 
empresa, del $5 \%$ al $7 \%$ del total de su venta. Considerando que ciertos porcentajes son otorgados a la banca privada, es necesario que se trabaje en nuevos sistemas de pagos, que brinden mayores beneficios a las empresas.

\section{d) A nivel internacional}

Debido a la inexistencia actual de una política de comercio integrado en la región de América Latina y El Caribe, a Ecuador se le dificultará la aplicación de un comercio electrónico transfronterizo como el B2B, motivo por el cual, deberá aprovechar al máximo los acuerdos comerciales a los que pertenezca y buscar alternativas como el ingreso a nuevos bloques comerciales. Evitar gravámenes gubernamentales por ventas a partir de plataformas electrónicas, será sin duda, un verdadero reto.

\section{Bibliografía}

Abebe, M. (2014). Electronic commerce adoption, entrepreneurial orientation and small- and medium-sized enterprise (SME) performance. Journal of Small Business and Enterprise Development, 21(1), 100-116.

Aguerre, C. (2019). Digital trade in Latin America: mapping issues and approaches. Digital Policy, Regulation and Governance, 21(1), 2-18.

Alsaad, A., Mohamad, R., Taamneh, A., \& Ismail, N. (2018). What drives global B2B ecommerce usage: an analysis of the effect of the complexity of trading system and competition pressure. Technology Analysis \& Strategic Management, 30(8), 1-13.

Alvarado, Á., \& Vergara, N. (2018). El desafío del comercio electrónico en la economía del Ecuador. Polo del conocimiento, 3(1), 67-86.

Ballesteros, D., \& Ballesteros, P. (2007). El comercio electrónico y la logística en el contexto latinoamericano. Scientia et Technica, 1(35), 269-274.

Barrientos, P. (2017). Marketing + internet = e-commerce: oportunidades y desafíos. Finanzas y Política económica, 9(1), 41-56.

Basantes, A., Gallegos, M., Guevara, C., Jácome, A., Posso, Á., Quiña, J., \& Vaca, C. (2016). Comercio electrónico. Ibarra: UTN. Obtenido de http://repositorio.utn.edu.ec/bitstream/123456789/6793/1/Libro\%20Final\%20E\%20com merce.pdf

Biemans, W., Brenčič, M., \& Malshe, A. (2010). Marketing-sales interface configurations in B2B firms. Industrial Marketing Management, 39(2), 183-194.

Broome, P. (2016). Conceptualizing the foundations of a regional e-commerce strategy: Open networks or closed regimes? The case of CARICOM. Cogent Business \& Management, 3(1), 1-32.

Campuzano, J., Chávez, G., \& Maza, J. (2018). El fracaso del dinero electrónico en Ecuador. $3 C$ Empresa: Investigación y pensamiento crítico, 7(3), 84-100.

Claver-Cortés, E., González-Illescas, M., Zaragoza-Sáez, P. C., \& Vargas Jiménez, M. (2017). Knowledge management in agricultural production associations. Revista Espacios, 38(52). Recuperado de http://www.revistaespacios.com/a17v38n52/17385221.html

Claver-Cortés, E., González-Illescas, M., Zaragoza-Sáez, P. C., \& Campuzano-Vázquez, J. (2017). Dimensiones de la dirección del conocimiento en las empresas productoras de camarón en Ecuador. Revista Espacios, 38(59). Recuperado de http://www.revistaespacios.com/a17v38n59/17385901.html 
CEPAL. (2018). Perspectivas del Comercio Internacional de América Latina y el Caribe. Naciones Unidas, Santiago. Obtenido de https://repositorio.cepal.org/bitstream/handle/11362/44196/5/S1801070_es.pdf

Chaudhuri, P., \& Kumar, A. (2015). Role of digitization and ecommerce in Indian economic growth: an employment generation perspective. Obtenido de Research Gate: https://www.researchgate.net/publication/291349502_Role_of_Digitization_and_Ecommerce_in_Indian_Economic_Growth_An_Employment_Generation_Perspective

Chen, H., Chen, S., \& Lan, Y. (2016). Attaining a sustainable competitive advantage in the smart grid industry of China using suitable open innovation intermediaries. Renewable and Sustainable Energy Reviews, 62, 1083-1091.

Chong, W., Bian, D., \& Zhang, N. (2015). E-marketing services and e-marketing performance: the roles of innovation, knowledge complexity and environmental turbulence in influencing the relationship. Journal of Marketing Management, 32(1-2), 149-178.

Costa, S., \& Elo, M. (2018). New Digital Layers of Business Relationships - Experiences From Business-To-Business Social Media. International Business in the Information and Digital Age, 13, 217-241.

Ecommerce Day. (2018). Ecommerce Day Ecuador. Obtenido de http://www.ecommerceday.ec/2018/el-evento/

El Comercio. (1 de abril de 2019). El comercio electrónico duplicó ventas en dos años. Obtenido de https://www.elcomercio.com/actualidad/comercio-electronico-ventas-ecuadorestudio.html

El Telégrafo. (21 de enero de 2019). E-Ecuador oferta en línea más de 2.000 productos ecuatorianos. Obtenido de https://www.eltelegrafo.com.ec/noticias/economia/4/eecuador-oferta-en-linea-mas-de-2-000-productos-ecuatorianos

El Universo. (3 de diciembre de 2017). $71 \%$ de cuentas de dinero electrónico, sin uso en Ecuador. Obtenido de https://www.eluniverso.com/noticias/2017/12/03/nota/6508306/71-cuentas-dineroelectronico-uso

El Universo. (14 de junio de 2018). e-Ecuador tiene 469 productos en línea. Obtenido de https://www.eluniverso.com/noticias/2018/06/14/nota/6809348/e-ecuador-tiene-469productos-linea

Erceg, A., \& Kilic, Z. (2018). Interconnection of e-commerce and logistics: examples from Croatia and Turkey. 18th International Scientific Conference Business Logistics in Modern Management, (págs. 265-283). Osijek. Obtenido de https://hrcak.srce.hr/ojs/index.php/plusm/article/view/7892

EU-Vietnam Business Network. (2018). E-commerce industry in Vietnam: Edition 2018. Ho Chi Minh, Vietnam. Obtenido de http://www.ukabc.org.uk/wpcontent/uploads/2018/09/EVBN-Report-E-commerce-Final-Update-180622.pdf

García, J. H. (2018). La regulación del comercio: retos ante el cambio tecnológico. Revista del Instituto de Ciencias Jurídicas de Puebla, 12(41), 43-70.

Gómez, M. (2018). América Latina: Informe sobre comercio electrónico 2018. Ecommerce Foundation.

Gutiérrez, E. (2015). Impacto del comercio electrónico en las grandes y medianas empresas de la ciudad de Sogamoso Boyacá, Colombia. Cuadernos Latinoamericanos de Administración, 11(20), 83-96. 
HaiBo, Y., Juan, L., \& Jie, L. (2016). Quantitative Analysis of E-commerce Application and Operation Performance in SMEs Based on Data Mining. International Journal of Database Theory and Application, 9(11), 149-162.

Hamad, H., Elbeltagi, I., Jones, P., \& El-Gohary, H. (2015). Antecedents of B2B E-commerce Adoption and its Effect on Competitive Advantage in Manufacturing SMEs. Strategic Change, 24(5), 405-428.

Internet World State. (2018). Internet Usage Statistics for all the Americas. Retrieved from https://www.internetworldstats.com/stats2.htm

Jones, C., Alderete, M., \& Motta, J. (2013). Adopción del comercio electrónico en Micro, Pequeñas y Medianas empresas comerciales y de servicios de Córdoba, Argentina. Cuadernos de Administración, 29(50), 164-175.

Jones, C., Motta, J., \& Alderete, M. (2016). Gestión estratégica de tecnologías de información y comunicación y adopción del comercio electrónico en Mipymes de Córdoba, Argentina. Estudios Gerenciales, 32(138), 4-13.

Karim, T., \& Qi, X. (2018). E-commerce development in Bangladesh. International Business Research, 11(11), 201-211.

Kim, H.-m., \& Lee, H.-h. (2016). Asset specificity and capability of e-Trade performance: evidence from Korea. Journal of Korea Trade, 20(1), 2-20.

Kshetri, N., \& Dholakia, N. (2002). Determinants of the Global Diffusion of B2B E-commerce. Electronic Markets, 12(2), 120-129.

Laudon, K., \& Guercio, C. (2017). E-commerce business. technology. society. Pearson.

Leonard, L., \& Jones, K. (2019). Trust in C2C Electronic Commerce: Ten Years Later. Journal of Computer Information 1-7. doi:https://doi.org/10.1080/08874417.2019.1598829

López, J., \& Jouanjean, M. (2017). Digital Trade: Developing a Framework for Analysis. OECD Trade Policy Papers(205), 13-14.

Mehta, D., \& Hamke, A. (2019, enero). In-depth: B2B eCommerce 2019. Statista Digital Market Outlook.

Meltzer, J. P. (2018, agosto). A digital trade policy for Latin America and the Caribbean. Banco Interamericano de Desarrollo.

Ministerio de Comercio Exterior e Inversiones. (abril de 2016). Más de 100 mipymes cuentan con herramientas de comercio electrónico. Obtenido de https://www.comercioexterior.gob.ec/mas-de-100-mipymes-cuentan-con-herramientasde-comercio-electronico/

Moertini, V. (2012). Small Medium Enterprises: On Utilizing Business-to-Business e-Commerce to Go Global. Procedia Economics and Finance, 4, 13-22.

Observatorio de Comercio Electrónico de la Universidad Espíritu Santo. (2018). Comportamiento de las transacciones no presenciales en Ecuador 2018. Universidad Espíritu Santo.

OMC. (2018). Informe sobre el comercio mundial 2018. OMC, Ginebra.

Pilinkiene, V., Kurschus, R., \& Auskalnyte, G. (2013). E-business as a source of competitive advantage. Economics and management, 18(1), 77-85.

Piñeiro, C., de Llano, P., \& Rodríguez, M. (2016). Las TIC como inductores de competitividad y facilitadores del éxito empresarial. International Journal of Information Systems and Software Engineering for Big Companies (IJISEBC), 3(1), 8-26.

Esta obra se comparte bajo la licencia Creative Common Atribución-No Comercial 4.0 International (CC BY-NC 4.0) Revista de la Universidad Internacional del Ecuador. URL: https://www.uide.edu.ec/ 
ProChile. (24 de junio de 2019). Ministerio de Relaciones Exteriores. Obtenido de ProChile presenta el primer sitio de eCommerce para exportadores: https://www.prochile.gob.cl/noticia/prochile-presenta-el-primer-sitio-de-ecommercepara-exportadores/

Ríos, A. (2014). Análisis y perspectivas del comercio electrónico en México. Enl@ ce: Revista Venezolana de Información, Tecnología y Conocimiento, 11(3), 97-121.

Sánchez, J. (2019). Plataformas e-Business. Anuario Jurídico y Económico Escurialense, 52, 307-334.

Seoane, E. (2005). La nueva era del comercio: El comercio electrónico. Las Tic al servicio de la gestión empresarial. Vigo: Ideaspropias. Obtenido de https://books.google.com.ec/books?id=evLz521ZVmAC\&printsec=frontcover\&hl=es\&so urce $=\mathrm{gbs} \_g e \_s u m m a r y \_r \& c a d=0 \# \mathrm{v}=$ onepage $\& \mathrm{q} \& \mathrm{f}=\mathrm{false}$

Sigmond, K. (2018). El comercio electrónico en los tratados de libre comercio de México. Revista IUS, 12(41), 359-377.

Sila, I. (2015). The state of empirical research on the adoption and diffusion of business-tobusiness e-commerce. International Journal of Electronic Business, 12(3), 258-301.

Silva, R. (2009). Beneficios del comercio electrónico. Perspectivas(24), 151-164.

Sojo, S. (2001). Comercio electrónico en Costa Rica. InterSedes: Revista de las Sedes Regionales, 2(2-3), 157-151.

Sukrat, S., Mahatanankoon, P., \& Papasratorn, B. (2016). The Evolution of C2C Social Commerce Models. Eleventh International Conference on Digital Information Management (ICDIM), 15-20. doi:https://doi.org/10.1109/ICDIM.2016.7829761

Suominen, K. (2017). Accelerating Digital Trade in Latin America and the Caribbean. Usina Digital de América Latina. Obtenido de LATE: https://late.online/wpcontent/uploads/2017/03/Late_Study_Full_ENG.pdf

Superintendencia de Bancos. (2018). Portal estadístico Superintendencia de Bancos. Obtenido de http://estadisticas.superbancos.gob.ec/portalestadistico/portalestudios/?page_id=1826

Tarazona, G., Medina, V. H., \& Giraldo, L. (2013). Modelo de implementación de soluciones de comercio electrónico. Revista Ingenierías Universidad de Medellín, 12(23), 131-143.

Teo, T., \& Ranganathan, C. (2004). Adopters and non-adopters of business-to-business electronic commerce in Singapore. Information \& Management, 42(1), 89-102.

Turban, E., King, D., Liang, T., Turban, D., \& Lee, J. (2015). Electronic Commerce: A Managerial and Social Networks Perspective. Springer. Obtenido de https://books.google.com.ec/books?id=pGxyBgAAQBAJ\&pg=PA56\&dq=types+e-

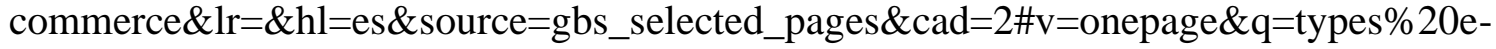
commerce $\& \mathrm{f}=$ false

Ueasangkomsate, P. (2015). Adoption E-Commerce for Export Market of Small and Medium Enterprises in Thailand. Procedia - Social and Behavioral Sciences, 207, 111-120.

UNCTAD. (2016). In Search of Cross-border E-commerce Trade Data: UNCTAD Technical Notes on ICT for Development $N^{\circ}$ 6. Obtenido de United Nations Conference on Trade and Development: https://unctad.org/en/PublicationsLibrary/tn_unctad_ict4d06_en.pdf

UNCTAD. (2017). Informe sobre la Economía de la información 2017: Digitalización, comercio y desarrollo. Naciones Unidas, Nueva York y Ginebra. Obtenido de https://unctad.org/es/PublicationsLibrary/ier2017_es.pdf

UNCTAD. (2018). Informe Sobre El Comercio Y El Desarrollo, 2018. Nueva York y Ginebra. Obtenido de https://unctad.org/es/PublicationsLibrary/tdr2018overview_es.pdf 
Winwin, M. (2019). The role of information technology in e-commerce. International Journal Of Scientific \& Technology Research, 8(1), 173-176.

Zatonatska, T., \& Kornieieva, T. (2016). Ukrainian e-commerce attractiveness: challenges and perspectives. Ekonomika, 95(1), 153-165.

Zwillenberg, P., Field, D., \& Dean, D. (2014). Greasing the Wheels of the Internet Economy. Obtenido de Boston Consulting Group: http://imagesrc.bcg.com/Images/Greasing_Wheels_Internet_Economy_Jan_2014_2_tcm9-90551.pdf 